\title{
ON THE CAPILLARY PRESSURE FUNCTION IN POROUS MEDIA BASED ON RELATIVE PERMEABILITIES OF TWO IMMISCIBLE FLUIDS
}

\author{
A.J. Babchin ${ }^{1}$ and B. Faybishenko ${ }^{2 *}$ \\ 1) Alberta Research Council, Edmonton, Canada, and Tel Aviv University, Israel, \\ 2) Lawrence Berkeley National Laboratory, Berkeley, California, USA. \\ ${ }^{*}$ Corresponding Author.
}

\begin{abstract}
The authors propose a new analytical approach and derive an explicit formula to determine a capillary pressure $\left(P_{c}\right)$ curve in porous media, by combining the first principles of surface science with the classical concept of the phase relative permeability. The developed formulae for $P_{\mathrm{c}}$ and a modified Leverett $J_{\mathrm{m}}$-function are based on the relative permeability functions for the wetting and nonwetting phases, an apparent specific surface area, and an apparent (calculated) contact angle $\Theta$. The application of the proposed approach was tested using several sets of data from existing publications. The developed $J_{\mathrm{m}}$-function can be described using the Weibull distribution model for drainage and imbibition conditions. The new approach can generally be used for any type of relative permeability functions and different types of wetting and nonwetting phases.
\end{abstract}

Key Words: Immiscible fluids, capillary pressure, relative permeability, specific surface area, porous media, Leverett J-function.

\section{Introduction}

Knowledge of reliable capillary pressure vs. saturation and relative permeability vs. saturation relationships is an important aspect in the field of numerical simulations of transport of nonaqueous phase liquids (NAPLs), including hydrocarbon liquids (oils), Dense NAPLs (DNAPLs), and 
Light NAPLS (LNAPs), in the subsurface. In their recent review of theoretical and experimental studies on the topic of spontaneous imbibition into porous and fractured media, Mason and Morrow [1] noted that there are fundamental problems with the application of differential equations for modeling, because of the nature and choice of appropriate relationships for the two (wetting and nonwetting) relative permeabilities and capillary pressure as functions of saturation. This issue has never been addressed in detail, and it is not clear how these functions should be determined using experiments $[1,2]$. Several studies showed that the use of a single effective average contact angle, $\Theta$, is not a physically correct approach for systems where there is a distribution of contact angles, especially in the event of the fractional wettability, nonwetting phase entrapment, and redistribution of nonwetting phase between different types of pores [3-9]. Direct measurement of two-fluid interfacial areas is difficult $[8,10]$.

In this paper, the authors propose to determine first an apparent specific surface area, using an explicit combination of the relative permeability functions for the wetting and nonwetting phases, which then will be used to assess the capillary pressure-saturation function. The results

of calculations using a new approach are demonstrated for the drainage and imbibition capillary pressure curves obtained using the data from [11-13].

\section{Theoretical Background of Proposed Approach}

Numerical simulations of transport phenomena of two immiscible fluids, such as NAPL and water, in porous media are usually based on using the Darcy law given by

$$
\begin{gathered}
q_{n}=-\frac{K K_{r n}}{\mu_{n}} \operatorname{grad} P_{n} \\
q_{w}=-\frac{K K_{r w}}{\mu_{w}} \operatorname{grad} P_{w}
\end{gathered}
$$

where $q_{\mathrm{n}}$ and $q_{\mathrm{w}}$ are the volumetric flow rates of the nonwetting and wetting phases, correspondingly, through the unit area, normal to flow; $K$ is the porous media absolute permeability; $K_{\mathrm{rn}}$ and $K_{\mathrm{rw}}$ are relative permeability of nonwetting and wetting phases, correspondingly; $?_{n}$ and $\Omega_{w}$ are viscosity of nonwetting and wetting phases, correspondingly, $P_{n}$ 
and $P_{\mathrm{w}}$ are pressures of nonwetting and wetting phases, and capillary pressure is given by $P_{\mathrm{c}}=P_{\mathrm{n}}-P_{\mathrm{w}}$.

For the purpose of simulation of flow of two immiscible fluids, $P_{\mathrm{c}}$ is often expressed by means of the Leverett-J function [14]. Purcell [15] introduced a relation between the permeability and capillary pressure. In his comments to the Purcell paper, Rose [16] presented an expression for relative permeability, using the fractional wetting phase saturation and Leverett's capillary pressure function. Burdine [17] introduced a tortuosity factor in the model describing the relationship between the relative permeability and capillary function, which for the case of twophase flow is given by

$$
\begin{gathered}
K_{r w}=\left(\lambda_{w}\right)^{2} \frac{\int_{0}^{S_{w} d S_{w}} / P_{c}{ }^{2}}{\int_{0}^{1 d S_{w} / P_{c}{ }^{2}}} \\
K_{r n}=\left(\lambda_{n}\right)^{2} \frac{\int_{S_{w}}^{1} d S_{n} / P_{c}{ }^{2}}{\int_{0}^{1 d S_{n} / P_{c}{ }^{2}}}
\end{gathered}
$$

where $\lambda_{w}$ and $\lambda_{n}$ are tortuosity factors, and symbols $w$ and $n$ denote the wetting and nonwetting phases, respectively, and $S_{w}$ and $S_{n}$ are saturations of the wetting and nonwetting phases, respectfully. However, the tortuosity factors are not explicitly defined and cannot be measured, thus, they are just fitting parameters.

Rapoport and Leas [18] were probably the first who suggested that the capillary pressure $\left(P_{\mathrm{c}}\right)$ and relative permeability functions are dependent on interfacial areas (although they have been typically modeled as functions of fluid saturation). In the past about 20-25 years, various numerical models and modeling techniques have been developed to simulate subsurface multiphase flow at the pore scale, such as pore network models and Lattice-Boltzmann models 
[19-22]. An application of a thermodynamically constrained macroscale description of flow in porous media, explicitly taking into account the presence of interfaces, was proposed in [23-24]. Despite the wide interest in measuring and calculating specific interfacial area and capillary pressure, there are surprisingly very few works on this subject [25].

Contrary to single-phase flow, when the specific surface area averaged over the total volume of a core sample, is a constant value, in the case of two-phase flow of immiscible fluids, the total specific surface area is a function of the phase distributions, and the total specific surface area is the sum of phase specific surface areas. The wetting phase tends to reside in smaller pores, while nonwetting fluid resides in larger pores. At residual wetting and nonwetting saturations the specific surface values asymptotically approach infinity. Indeed, the residual wetting phase is located in the smallest capillaries, as tiny ganglia, or as pendular drops, bridging the solid grains. All these configurations have very small radii, and, thus, high capillary pressure, exhibiting high capillary resistance to flow, which the pressure gradient, imposed by displacement fluid flow, is incapable to overcome. Complete immobilization of the residual fluid is either equivalent to apparent radii approaching zero value, or to specific surface approaching infinity. In other words, capillarity effects should be taken into account in the evaluation of the specific surface area for a two-phase flow system in porous media, as these effects induce additional resistance to flow. Even in simplified droplet train model, capillary forces are capable of inducing additional resistance to flow [26].

Babchin and Nasr [27] derived a simplified analytical expression for the capillary pressure gradient in homogeneous porous two-fluid media, containing three bulk phases (solid phase, wetting and nonwetting fluids), with three possible interfaces (solid-wetting, solid-nonwetting, and nonwetting-wetting). They assumed a finite value of the three-phase contact angle between two fluids and a solid phase of porous rock, and that the contact surface area between two continuous fluid phases is small in comparison with contact surface areas between each fluid and porous rock in the unit volume of the rock. The surface energy of such a system can be given by 


$$
W=\gamma_{n-s} \cdot S_{v} \cdot S_{n}+\gamma_{w-s} \cdot S_{v} \cdot S_{w}+\gamma_{n-w} \sigma_{n-w}
$$

where $W$ is the density of excess energy of porous media associated with interfaces, and in other words, it is the additional density of surface energy, due to the presence of two fluids within porous rock, $S_{v}$ is the specific surface area of porous media, $\gamma_{n-s}, \gamma_{w-s}$ and $\gamma_{n-w}$ are nonwetting phase-solid, wetting phase-solid, and nonwetting-wetting phases specific surface energies, respectively, $S_{n}$ and $S_{w}$ are saturations of nonwetting liquid and water, subject to $S_{n}+$ $S_{\mathrm{w}}=1$, and $\sigma_{\mathrm{n}-\mathrm{w}}$ is area of nonwetting-wetting phases interface within unit volume of porous media.

According to [27], the area of direct contact between two immiscible fluids in a one-Darcy permeability porous media is five orders of magnitude smaller than the area of contact between these fluids and solid grains, therefore the last term of Eq. (2) can be neglected. (For solid-liquid and liquid-liquid contact areas to be of the same order, one fluid should be emulsified in another, and droplet size should be of the same order of magnitude as the solid grain size. Such a state is known as emulsion flow, when one of the fluids is in discontinuous state, and no three phase contact angle exist. This kind of flow is rarely observed and is outside the scope of this paper.)

Equation (2) can then be given by

$$
W \approx\left(\gamma_{n-s}-\gamma_{w-s}\right) \cdot S_{v} \cdot S_{n}+\gamma_{w-s} \cdot S_{v}
$$

Using the standard Young equation, $\gamma_{n-s}-\gamma_{w-s}=\gamma_{n-w} \cos \Theta$, where $\Theta$ is wetting-phase contact angle, we can express $W$ as

$$
W \approx \gamma_{n-w} \cdot \cos \Theta \cdot S_{v} \cdot S_{n}+\gamma_{w-s} \cdot S_{v}
$$


The second term in Equation (4) corresponds to the condition of the full water saturation of the porous space, when $S_{w}=1$. The first term of Eq. (4) represents the energy arising from the capillary pressure, which is developed in the system of two immiscible fluids in porous media.

$$
P_{c} \approx \gamma_{\mathrm{n}-\mathrm{w}} \cdot \cos \Theta \cdot \mathrm{S}_{\mathrm{v}} \cdot \mathrm{S}_{\mathrm{n}}
$$

The value of $S_{v}$ in Eq. (5) can be expressed as an averaged value for a volume $V$ of porous media in the following integral form:

$$
S_{V}=\frac{1}{V} \int_{o}^{V} \overline{S_{v}} d V
$$

The value $\overline{S_{v}}$ in Eq. 6 can be presented as a sum of two components-one for nonwetting, the other for wetting phases, both being saturation dependent. For a case of a single fluid residing in porous media, the averaged pore size can be expressed in term of the equivalent pore radius, $r_{e}$, using (a) the apparent specific surface $S_{\mathrm{v}}$ [note that for a single fluid, according to Eq. (6), one obtains $S_{v}=\overline{S_{v}} ;$ for two-phase flow, see Eqs. (10) and (11)]

$$
r_{e}=1 / S_{\mathrm{v}}
$$

and (b) porous media permeability, $K$, and porosity, $\phi$, given by

$$
r_{e}=(K / \phi)^{1 / 2}
$$

By equating Eqs. (7a) and (7b), the relationship for the averaged $S_{v}$ is given by

$$
S_{v}=(\phi / K)^{1 / 2}
$$


In the case of transport of two immiscible fluids in porous media, the permeabilities of nonwetting and wetting phases, subject to $S_{n w}+S_{w}=1$, can be expressed in terms of their relative permeabilities, which are functions of phase saturations, given by

$$
\begin{aligned}
& K_{\mathrm{nw}}=K K_{\mathrm{rn}}\left(S_{\mathrm{nw}}\right) \\
& K_{\mathrm{w}}=K K_{\mathrm{rw}}\left(S_{\mathrm{w}}\right)
\end{aligned}
$$

Using Eqs. (8) and (9), and assuming a constant porosity, [? we can define the equations to describe the relationships between the apparent specific surface area and permeability of each phase:

$$
\begin{aligned}
& \overline{S_{v \mathrm{n}}}=\left[\phi /\left(K K_{\mathrm{rn}}\right)\right]^{1 / 2} \\
& \overline{S_{v \mathrm{w}}}=\left[\phi /\left(K K_{\mathrm{rw}}\right)\right]^{1 / 2}
\end{aligned}
$$

The total apparent specific surface, averaged within the volume $V$, and which is a function of the liquid phases saturation, can be expressed as the sum of Eqs. (10a) and (10b) given by:

$$
\begin{aligned}
& S_{\mathrm{v}}=\overline{S_{v \mathrm{n}}}+\overline{S_{v \mathrm{w}}}=\phi^{1 / 2}\left[\left(1 / K K_{\mathrm{rn}}\right)^{1 / 2}+\left(1 / K K_{\mathrm{rw}}\right)^{1 / 2}\right] \\
& \text { or } \\
& S_{\mathrm{v}}=(\phi / K)^{1 / 2}\left(K_{\mathrm{rnw}}{ }^{1 / 2}+K_{\mathrm{rw}}{ }^{1 / 2}\right) /\left(K_{\mathrm{rn}} \cdot K_{\mathrm{rw}}\right)^{1 / 2}
\end{aligned}
$$

At the residual saturation points $S_{\mathrm{rn}}$ and $S_{\mathrm{rw}}$ for nonwetting and wetting phases, respectfully, we have $K_{\mathrm{rn}}=0$ and $K_{\mathrm{rw}}=0$, and the solution of Eq. (11) will result become singular (i.e, a function that is not finite or differentiable). The singularity at $K_{\mathrm{rw}}=0\left(\mathrm{at} s=s_{\mathrm{rw}}\right)$ corresponds to the wellknown phenomena of an infinite suction pressure at the irreducible wetting-phase saturation 
[28]. The physical explanation of these phenomena is as follows: at the residual saturation points, the fluid phase resides in tiny pores or in the form of bridges matching the grains or tiny ganglia, all of them having very small radius, rendering very high capillary pressure, resisting either displacement or imbibition process beyond residual saturation points. For example, the residual oil saturation is defined, generally, as the oil content that remains in an oil reservoir at depletion, when oil ceases to be recovered [29]. The residual oil saturation in the reservoir can range from $2 \%$ to $50 \%$, with an approximate average from $15 \%$ to $20 \%$ [30]. Note that the results of laboratory and field tests are often inconsistent and lead to the uncertainty in the values of the oil residual saturation [31].

To remove infinite values upon calculations, we can employ the system of reduced (normalized) saturations $s_{\mathrm{n}}$ and $s_{\mathrm{w}}$ for nonwetting and wetting phases, respectively given by [11]:

$$
\begin{aligned}
& s_{n}=\left(s_{n}-s_{r n}\right) /\left(1-s_{r n}-s_{r w}\right) \\
& s_{w}=\left(s_{w}-s_{r w}\right) /\left(1-s_{r n}-s_{r w}\right)
\end{aligned}
$$

Expressing nonwetting phase saturation in the reduced (normalized) form, we finally come to the following expression for the nonwetting phase-water-solids capillary pressure:

$$
P_{\mathrm{c}}=\gamma_{\mathrm{nw}} \cos \theta \cdot S_{\mathrm{v}} \cdot s_{\mathrm{n}}+A
$$

where $S_{v}$ is given by Eq. (11), $S_{n}$ is given by Eq. (12), $A$ is a constant that corresponds to initial displacement pressure, when wetting phase is displaced by nonwetting phase, and $A=0$ for imbibition [11]. Thus, based on Eq. (13), intrinsic hysteresis of $P_{\mathrm{c}}$ for drainage (water is displaced 
by nonwetting phase) and imbibition (nonwetting phase is displaced by water) conditions is determined within the constant.

\section{Application of the Proposed Approach and Results of Calculations}

The proposed approach was tested using a comparison of the results of calculations of $P_{\mathrm{c}}$ with published data given by Collins [11], Das et al. [12], Beckner et al. [13, 32]. The input parameters for calculations of relative permeability and $P_{\mathrm{c}}$ curves, which were taken from these publications, are summarized in Table 1. (The graphs plotted in the referenced publications were digitized using the Plot Digitizer, Version 2.6.4, a Java program written by J.A. Huwaldt http://plotdigitizer.sourceforge.net )

Figures 1 through 4 depict the relative permeability curves (upper figures), apparent specific areas vs. saturation (middle figures) calculated from Eq. (11), and the resulting relationships $P_{\mathrm{c}}$ vs. $S_{w}$ (lower figures), calculated from Eq. 13. In calculations of the drainage curves, we used constant values of $A$ that equal to the capillary pressure at the residual saturation point $S_{n r}$, and for the imbibition curves, $A=0$. The match between the calculated and experimental $P_{\mathrm{c}}$ curves was achieved using the contact angle, as a fitting parameter, given in Table 1. Note that in all cases, the calculated contact angles are $0<\Theta<90^{\circ}$, which is indicative of water-wet conditions. (The fact of relatively high-calculated values of $\Theta$ will be addressed in another paper by the authors.) The fact of the calculated contact angle $\Theta>0$ could be explained by the fractional wettability of porous media $[3,33]$. Based on the results of calculations given in Table 1, fractional wettability (determined as $\cos \Theta$ ) varies from 0.05 to 0.31 . The correlation analysis 
showed no correlation of the calculated apparent contact angle $\Theta$ with the absolute permeability, porosity, and specific surface area.

The results of $P_{\mathrm{c}}$ calculations from Eq. (13) were then used to evaluate a modified Leverett $J_{m^{-}}$ function given by

$$
J_{m}\left(S_{w n}\right)=\frac{P_{c}}{\gamma_{n w} \cos \Theta} \sqrt{\frac{K}{\varnothing}}
$$

where $\cos \Theta$ is determined using the contact angle $\Theta$ given in Table $1, P_{c}$ is taken in Bars, absolute permeability $K$ is in $\mathrm{m}^{2}$, and $\gamma_{n-w}$ is in $\mathrm{N} / \mathrm{m}$, and the values of normalized wetting phase saturation $s_{w}$ are determined from Eq. (12b).

Combining Eqs. (13) and (14), we obtain a new form of the modified Leverett function given by

$$
J_{m}\left(S_{w}\right)=\frac{P_{c}}{P_{c}-A} S_{v} S_{n} \sqrt{\frac{K}{\varnothing}}
$$

For the imbibition curve, when $A=0$, Eq. (15) can be simplified to

$$
J_{m}\left(s_{w}\right)=S_{v} s_{n} \sqrt{\frac{K}{\varnothing}}
$$

Figure 5 of the modified Leverett function $\left(J_{m}\right)$ vs. the normalized wetting saturation $\left(s_{w}\right)$ shows that despite a significant difference in absolute and relative permeabilities and capillary pressure curves shown in Figures 1 though 4 , the scattering of points around the modified Leverett $J_{\mathrm{m}}\left(s_{\mathrm{w}}\right)$ function is minimal. 
Statistical analysis of the calculated $P_{c}$ values of shown in Figures 1 through 4 , shows that the modified Leverett $J_{m}\left(s_{w}\right)$ function can be described using a 4-parameter Weibull distribution model given by

$$
J_{m}=a-b \cdot \exp \left(-c s_{w}^{d}\right)+A
$$

with the following coefficients: $a=0.000589, b=0.000582, c=0.02208$, and $d=-0.80742213$. The value of $A=1.98 \mathrm{E}-5$ in Eq. (17) for drainage, and $A=0$ for imbibition. (The coefficient of correlation of fitting of Eq. (17) to the experimental data shown in Figure 5 is 0.98.) Note that the Weibull distribution model is one of the most widely used statistical distribution models in reliability engineering and statistical data analysis due to its versatility.

\section{Conclusions}

Based on the notion of the specific surface area, the authors presented an analytical approach to determine the capillary pressure curve and a modified Leverett $J_{\mathrm{m}}$ - function of two immiscible fluids in porous media, using a combination of the relative permeability functions for the wetting and nonwetting phases along with an equation for an apparent specific surface area and an apparent contact angle. Based on the Leverett $J_{\mathrm{m}}$-function for $P_{\mathrm{c}}$ in terms of relative permeability, a solution for unstable fingering front of heavy oil displacement by water, considered [34], can be solved analytically.

In this paper, the results of calculations of $P_{\mathrm{c}}$ were used to assess the values of the contact angle $\Theta$, which was then applied to assess the modified Leverett $J_{m}$-function versus the normalized wetting phase saturation. The $J_{\mathrm{m}}$-function is described using the Weibull distribution model for both the drainage and imbibition conditions. 
Acknowledgement: The work of the $2^{\text {nd }}$ author was partially supported by the Sustainable Systems Scientific Focus Area (SFA) program at LBNL, supported by the U.S. Department of Energy, Office of Science, Office of Biological and Environmental Research, Subsurface Biogeochemical Research Program, through Contract No. DE-AC02-05CH11231 between Lawrence Berkeley National Laboratory and the U. S. Department of Energy. The authors are very much thankful to Dr. Christine Doughty of LBNL and an anonymous reviewer for their careful review and valuable comments, which helped the authors to improve the manuscript. The authors would also like to thank Prof. Jacob Bear and Prof. Grigory Issakovich Barrenblatt for reading the original manuscript and their opinion that the paper is interesting and deserves publication.

\section{References}

[1] Mason J., and N. Morrow, Developments in spontaneous imbibition and possibilities for future work, Journal of Petroleum Science and Engineering. 110 (2013) 268-293, 2013

[2] Larson, R.G., and N.R. Morrow, Effects of Sample Size on Capillary Pressures in Porous Media, Powder Technology. 30 (1981) 123-138.

[3] Bradford, S.A., and F.J. Leij, Fractional wettability effects on two-and three-fluid capillary pressure-saturation relations, Journal of Contaminant Hydrology 20 (1995) 89-109.

[4] van Dijke, M.I.J.. K.S. Sorbie, S.R. McDougall, Saturation-dependencies of three-phase relative permeabilities in mixed-wet and fractionally wet systems, Adv in Water Resources 24 (2001) 365-384.

[6] Behbahani, H.Sh., Blunt, M.J., Analysis of imbibition in mixed wet rocks using pore-scale modelling. SPE 90132, Annual Technical Conference and Exhibition, Houston, Texas, USA. (2004).

[7] Behbahani, H. Sh., G.Di Donato, M.J. Blunt, Simulation of counter-current imbibition in waterwet fractured reservoirs, Journal of Petroleum Science and Engineering 50 (2006) 21- 39.

[8] Schramm, L.L, Surfactants: Fundamentals and Applications in the Petroleum Industry, Cambridge University Press, 2010.

[9] Raeesi, B., N.R. Morrow, G. Mason, Effect of surface roughness on wettability and displacement curvature in tubes of uniform cross-section, Colloids and Surfaces $A$ : Physicochemical and Engineering Aspects, Colloids and Surfaces A: Physicochem. Eng. Aspects 436 (2013) 392- 401.

[10] O'Carroll, D.M., L.M. Abriola,T, Catherine A. Polityka, S.A. Bradford, A.H. Demond, Prediction of two-phase capillary pressure-saturation relationships in fractional wettability systems, Journal of Contaminant Hydrology 77 (2005) 247- 270

[11] Collins, R.E., Flow of Fluids through Porous Materials, Reinhold Publishing Corporation, New 
York. 1961.

[12] Das, D. B., S. M. Hassanizadeh, B.E. Rotter and B.Ataie-Ashtiani, A Numerical Study of Microheterogeneity Effects on Upscaled Properties of Two-phase Flow in Porous Media, Transport in Porous Media 56 (2004) 329-350.

[13] Beckner, B. L., A. Firoozabadi, K. Aziz, Modeling transverse imbibition in double-porosity simulators, paper presented at SPE California Regional Meeting, Long Beach, Calif., 23-25 March. 1988.

[14] Bear, J., Dynamics of Fluids in Porous Media, Dover Publications, 1972.

[15] Purcell, W. R., Capillary pressures - Their measurement using mercury and the calculation of permeability, Trans. AIME, 186 (1949) 39-46.

[16] Rose, W. Comments to the paper by Purcel, Trans. AIME, 186 (1949) 46-48.

[17] Burdine, N. T., Relative permeability calculations from pore size distribution data, Trans. AIME, 198 (1953) 71.

[18] Rapoport, L.A., Leas, W.J., 1951. Relative permeability to liquid in liquid-gas systems. Trans. Am. Inst. Mineral. Metall. Petrol. Eng. 192, 83-95.

[19] Reeves P. and M. Celia. A functional relationship between capillary pressure, saturation, and interfacial area as revealed by a pore-scale network model. Water Resources Research, 32 (1996), 2345-2358,

[20] Held P. and M. Celia. Modeling support of functional relationships between capillary pressure, saturation, interfacial area and common lines. Advances in Water Resources, 24 (2001) 325-343.

[21] Culligan, K.A., D.Wildenschild, B.S. Christensen, W.G. Gray, M.L. Rivers, and A.F.B.Tompson, Interfacial area measurements for unsaturated flow through a porous medium, Water Resour. Res., 40 (2004), W12413.

[22] Niessner J. and S.M. Hassanizadeh. A Model for Two-Phase Flow in Porous Media Including Fluid-Fluid Interfacial Area. Water Resour. Res., 44 (2008) W08439.

[23] Gray, W. G., and S. M. Hassanizadeh (1989), Averaging theorems and averaged equations for transport of interface properties in multiphase systems, Int. J. Multiphase Flow, 15, 8195.

[24] Gray, W. G., A. F. B. Tompson, and W. E. Soll, Closure conditions for two-fluid flow in porous media, Transp. Porous Media, 47 (2002) 29-65.

[25] Joekar-Niasar, V., S. M. Hassanizadeh, and A. Leijnse. Insights into the relationship among capillary pressure, saturation, interfacial area and relative permeability using pore-scale network modeling. Transport in Porous Media, 74 (2008) 201-219.

[26] Babchin and J.-Y Yuang, On the Capillary Coupling between Two Phases in a Droplet Train Model, Transport in Porous Media, 26 (1997) 225-228.

[27] Babchin, A.J., T.N. Nasr, Analytical Model of the Capillary Pressure Gradient in Oil-Water-Rock System, Transport in Porous Media, 65 (2006) 359-362.

[28] Brooks, R.H., and Corey, A.T., Hydraulic Properties of Porous Media, Hydrol. Pap.3. Colorado St ate University, Fort Collins (1964).

[29] Bradley, H.B., Petroleum Engineering Handbook, 1st edition, Society of Petroleum Engineers, 1987. ISBN 1-55563-010-3.

[30] Morrow, N.R., A Review of the Effects of Initial Saturation, Pore Structure and Wettability on Oil Recovery by Waterflooding, In Proc. North Sea Oil and Gas Reservoirs Seminar, 
Trondheim (December 2-4, 1985), Graham and Trotman, Ltd., London (1987) 179 -191. [31] Donaldson, E.C., G.V. Chilingarian, T.F. Yen, Enhanced Oil Recovery, II: Processes and Operations, Elsevier, 1989.

[32] Li K. and R.N. Horne, Comparison of methods to calculate relative permeability from capillary pressure in consolidated water-wet porous media, Water Resour. Res., 42 (2005) W06405.

[33] Boinovich, L. and A.M. Emelyanenko, The Analysis of the Parameters of Three-phase Coexistence in the Course of Long-term Contact between a Superhydrophobic Surface and an Aqueous Medium, Chem. Lett. 41 (2012).

[34] Babchin, A., I.Brailovsky, P.Gordon, and G.Sivashinsky, On Fingering Instability in Immiscible Displacement, Physical Review E, 03 (2008) 77. 
Table 1. Parameters used for calculations of $P_{\mathrm{c}}$.

\begin{tabular}{|c|c|c|c|c|}
\hline Parameters & Das et al. (2004) & $\begin{array}{c}\text { Beckner et al. (1988) } \\
\text { (cited in Li and } \\
\text { Horne, 2006, Fig.9) }\end{array}$ & $\begin{array}{c}\text { Collins (1961, } \\
\text { Fig. 6-7) }\end{array}$ & $\begin{array}{c}\text { Collins (1961, } \\
\text { Fig. 6-13) }\end{array}$ \\
\hline Porosity, $\varnothing$ & 0.4 & 0.225 & 0.225 & 0.32 \\
\hline Permeability, $K\left(\mathrm{~m}^{2}\right)$ & $5.00 \mathrm{E}-12$ & $2.90 \mathrm{E}-12$ & $2.90 \mathrm{E}-12$ & $1.97 \mathrm{E}-13$ \\
\hline Surface tension, $\gamma_{n w}(\mathrm{~N} / \mathrm{m})$ & 0.02 & 0.048 & 0.03 & 0.048 \\
\hline $\mathrm{S}_{\mathrm{rn}}$ & 0.92 & 0.75 & 0.75 & 0.85 \\
\hline $\mathrm{S}_{\mathrm{rw}}$ & 0.098 & 0.346 & 0.3 & 0.092 \\
\hline$A$ (bars) & 0.0135 & 0.04 & 0 & 0.1 \\
\hline $\begin{array}{c}\text { Calculated apparent } \\
\text { contact angle } \\
\Theta(\mathrm{deg})\end{array}$ & 87 & 80 & 72 & 84 \\
\hline Nonwetting fluid & PCE & oil & oil & oil \\
\hline
\end{tabular}




\section{Figure Captions}

Figure 1. Comparison of calculated $P_{\mathrm{c}}$ curves with data from the paper by Das et al. [12]: upper figurerelative permeability curves (solid curve-wetting phase, dashed curve-nonwetting phase), middle figure-calculated specific surface area, and lower figure-calculated $P_{\mathrm{c}}$ curves: symbols are the values from [12], and a solid line is a calculated $P_{\mathrm{c}}$ curve). Left vertical dashed line -residual wetting phase saturation, and right vertical dashed line-nonwetting phase residual saturation. Note: in all figures the $x$ axis is the wetting phase saturation, $S_{\mathrm{w}}$.

Figure 2. Comparison of calculated $P_{\mathrm{c}}$ curves with data from [13] (as given in [32]: upper figure-relative permeability curves (solid curve-wetting phase, dashed curve-nonwetting phase), middle figurecalculated specific surface area, and lower figure-calculated Pc curves: symbols are from Das et al., 2004, and a solid line is a calculated $P_{\mathrm{c}}$ curve).

Figure 3. Comparison of calculated $P_{\mathrm{c}}$ curves with data from Figure 6-7 of the book by Collins [11]: upper figure-relative permeability curves (solid curve-wetting phase, dashed curve-nonwetting phase), middle figure-calculated specific surface area, and lower figure-calculated $P_{c}$ curves: symbols are from [11], and a solid line is a calculated $P_{\mathrm{c}}$ curve).

Figure 4. Comparison of calculated Pc curves with data from Figure 6-13 of the book by Collins [11]: upper figure-relative permeability curves (solid curve-wetting phase, dashed curve-nonwetting phase), middle figure-calculated specific surface area, and lower figure-calculated Pc curves: symbols are from Collins [11], and the solid and dashed lines are the calculated $P_{\mathrm{c}}$ curves for the drainage and imbibition).

Figure 5. Modified Leveret $J_{\mathrm{m}}$-function calculated using the $P_{\mathrm{c}}$ from Eq.(13), with $K, \phi$, and $\Theta$ given in Table 1 vs. normalized saturation calculated from Eq. (12b), and fitting curves for the drainage and imbibition described by Eq. (17). 

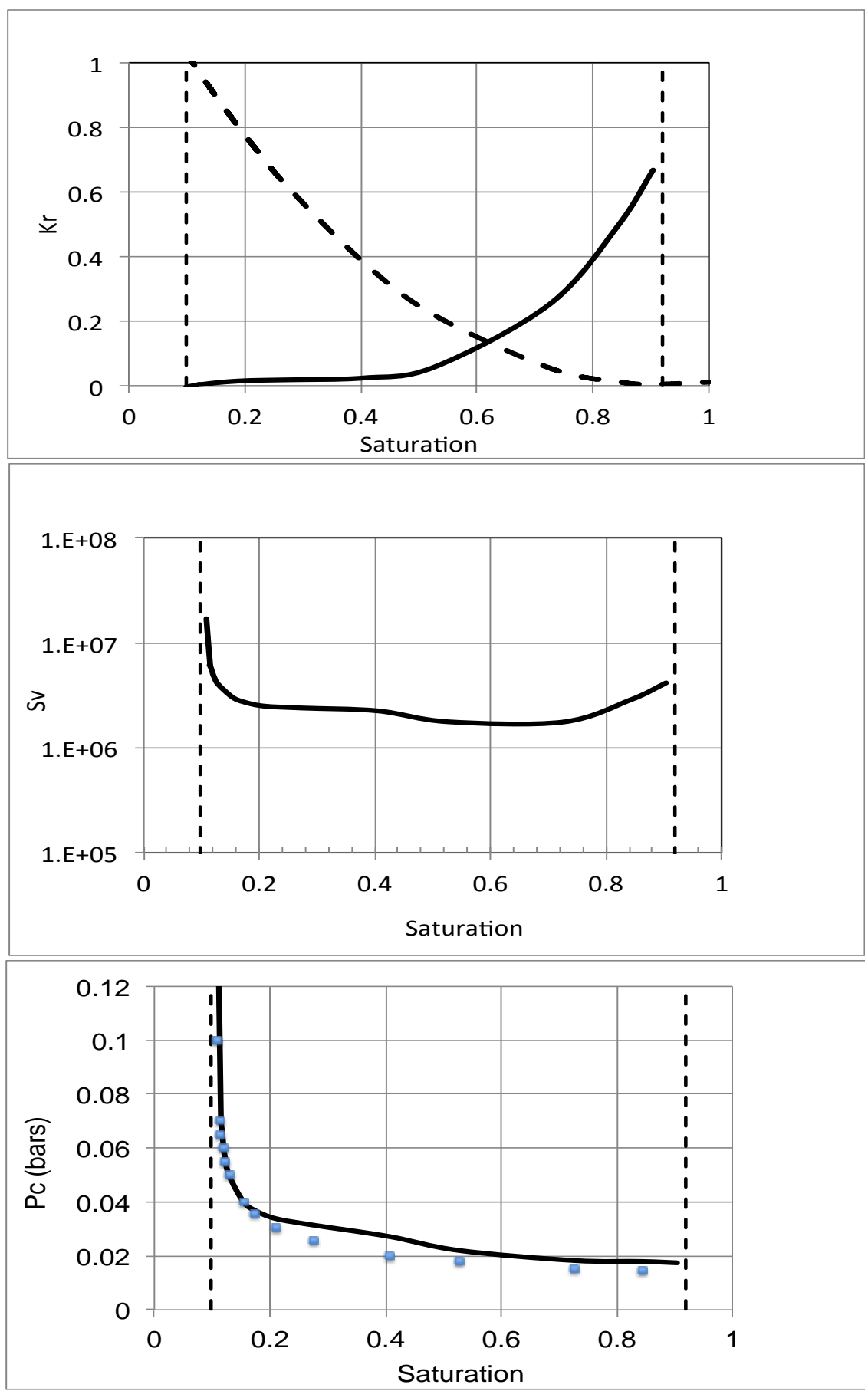

Figure 1. 

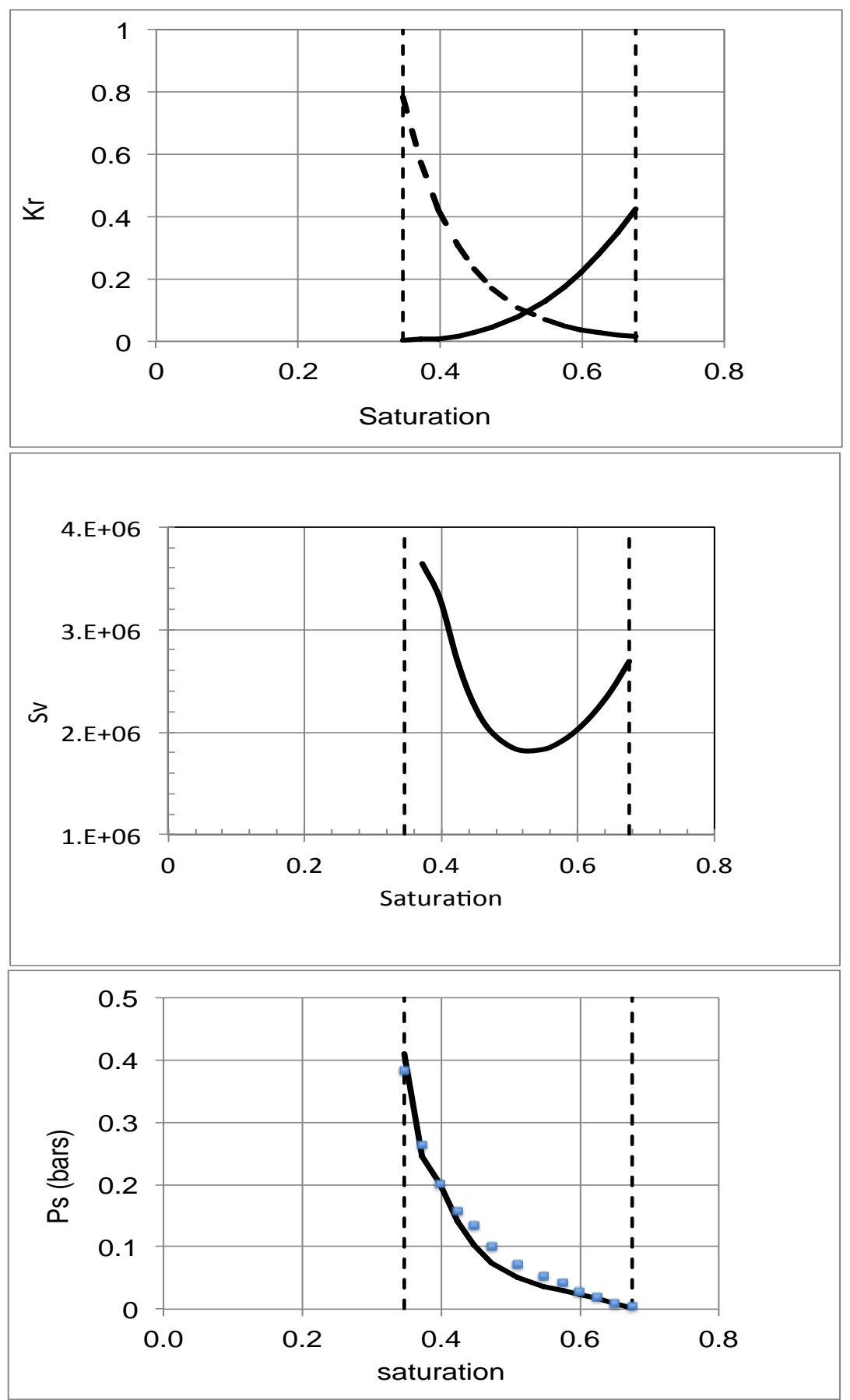

Figure 2 . 

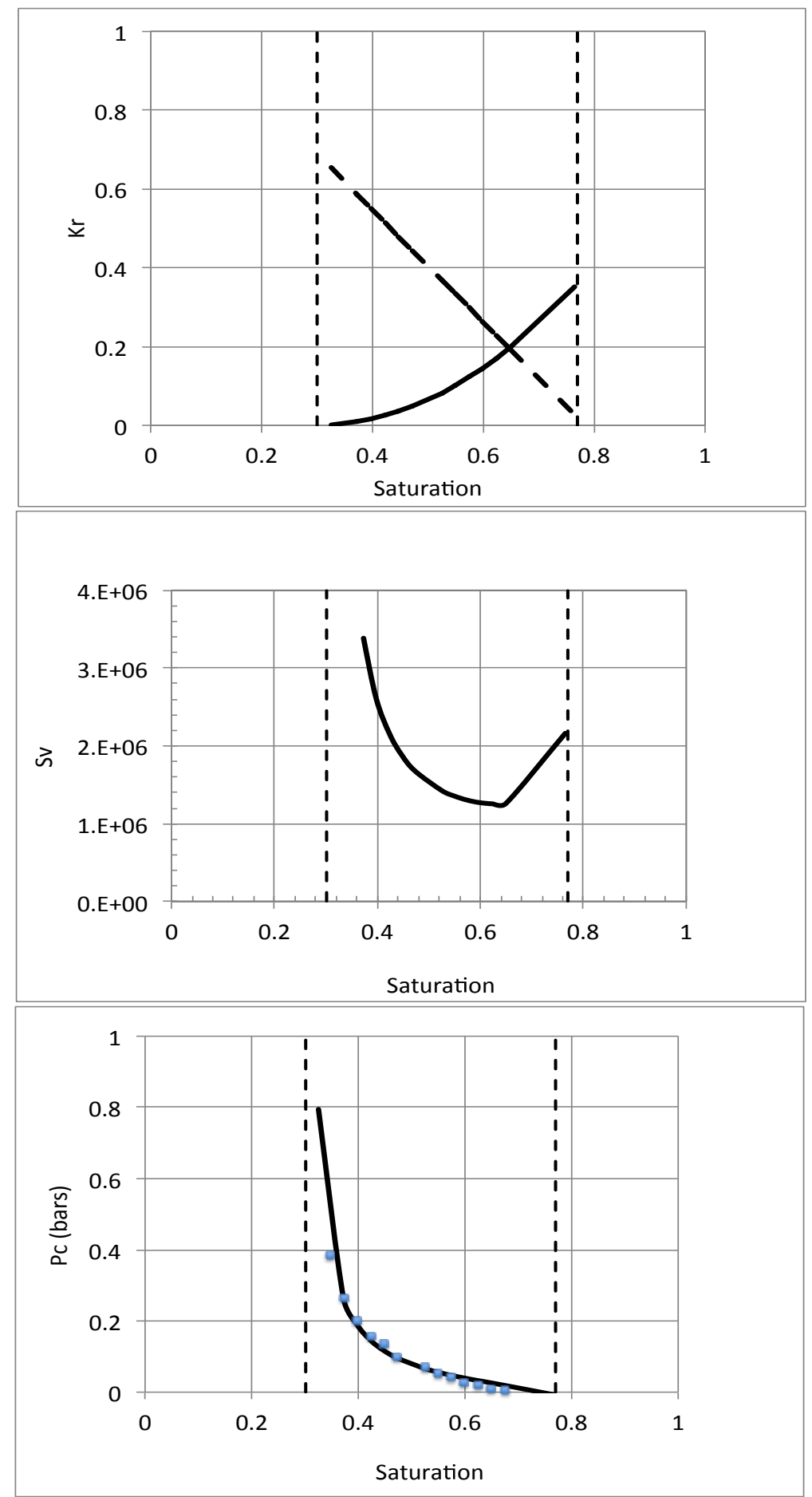

Figure 3. 

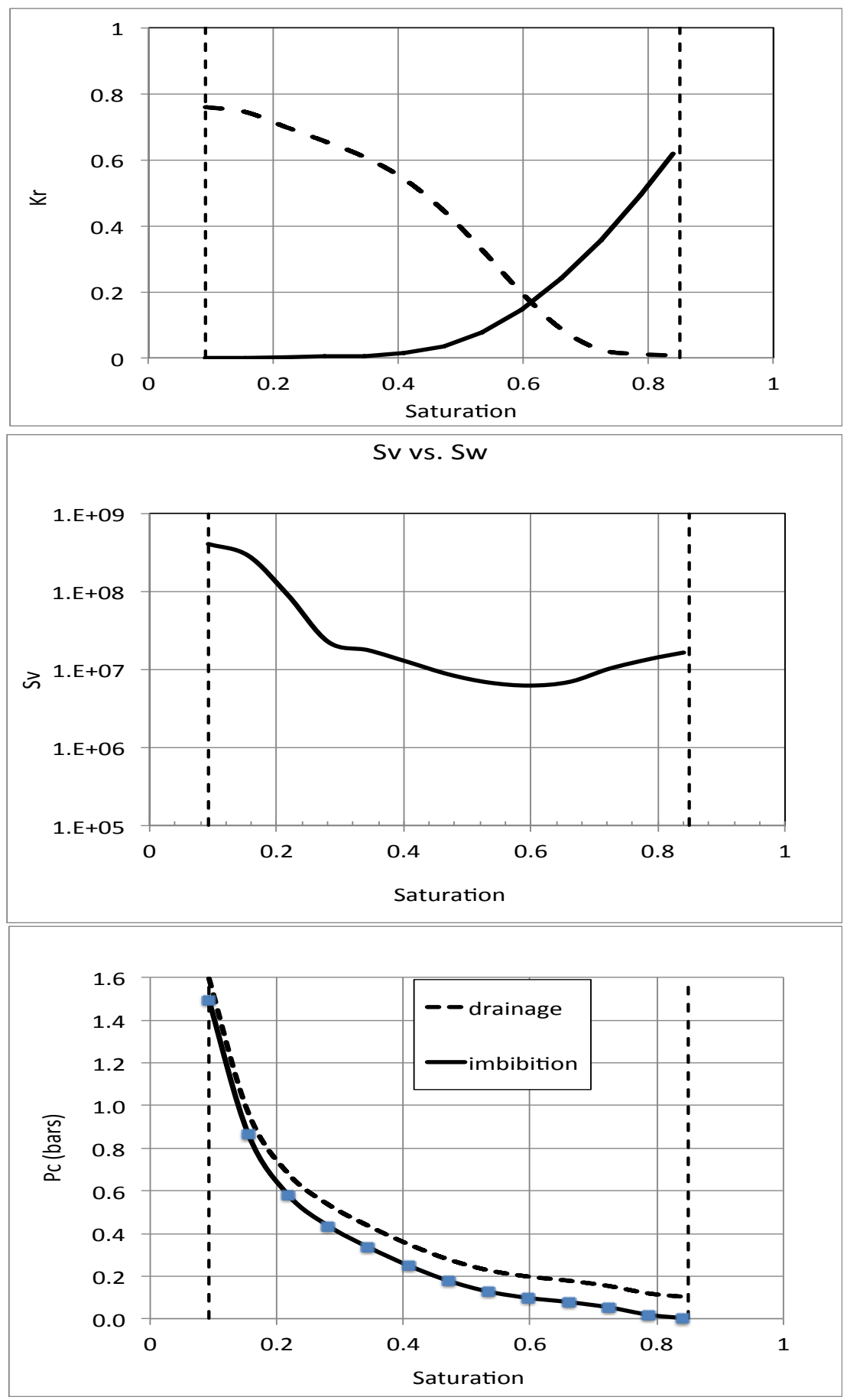

Figure 4. 


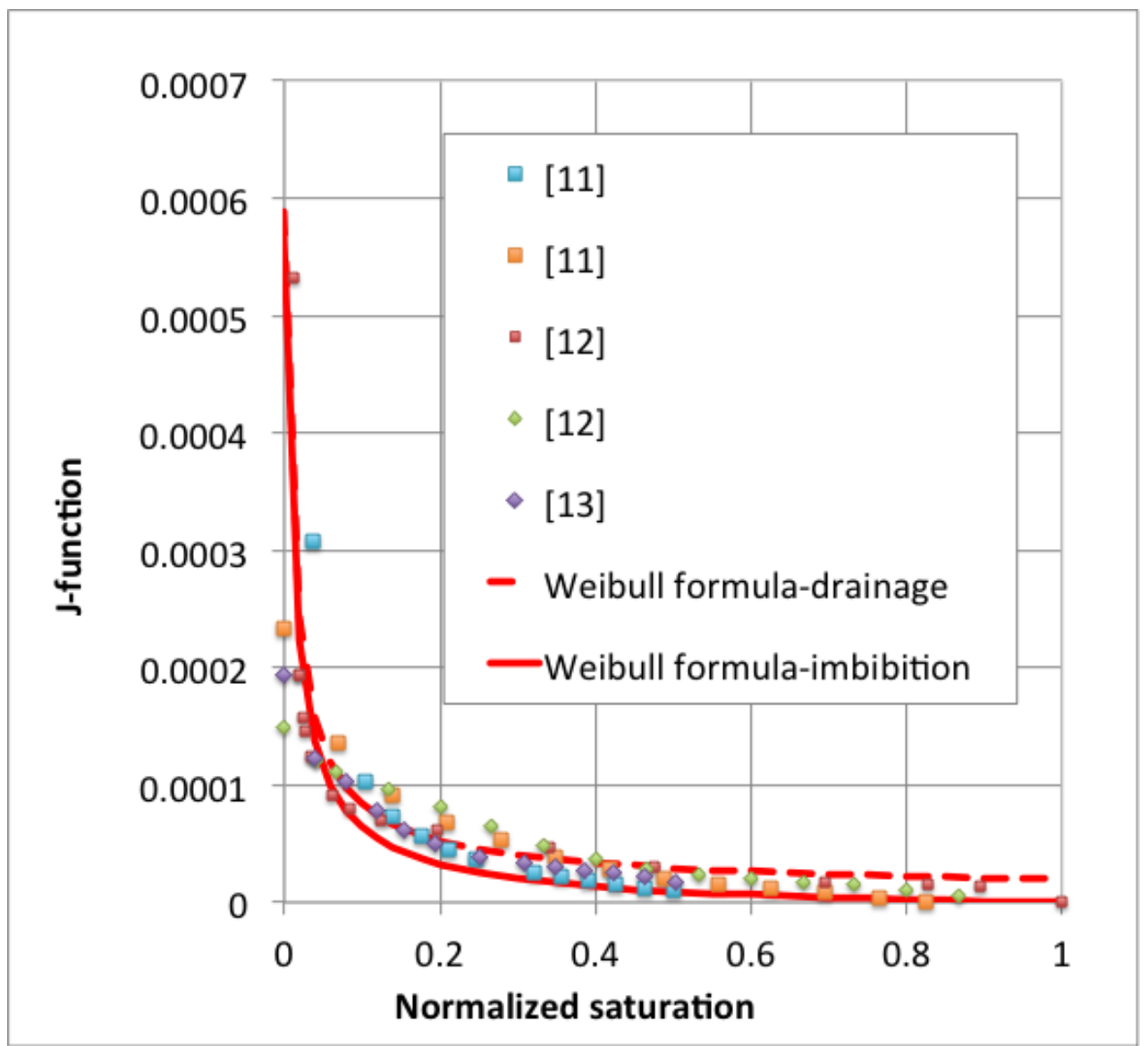

Figure 5. 


\section{Modified $J$-Leverett function calculated from relative permeabilities}

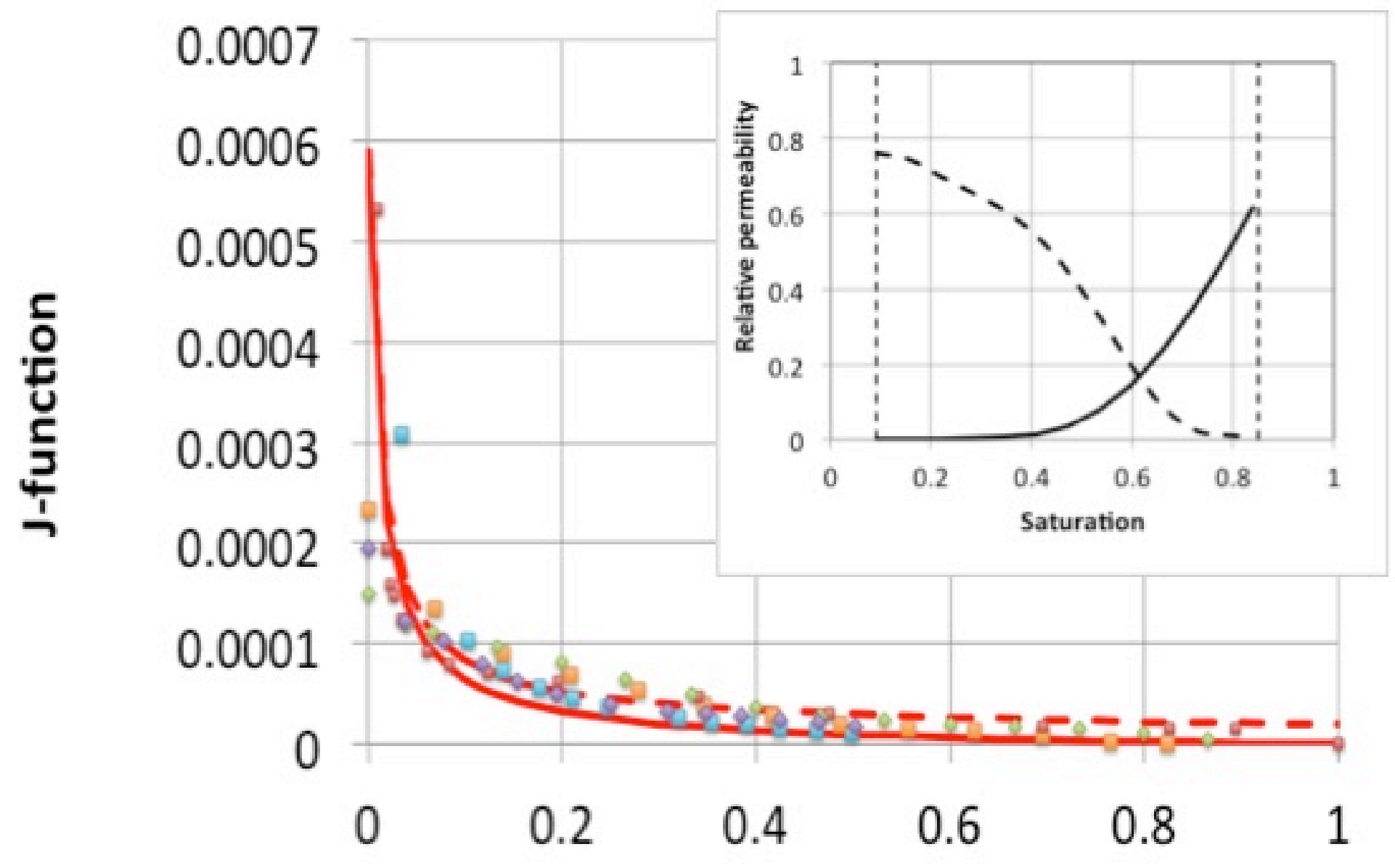

Normalized saturation 